
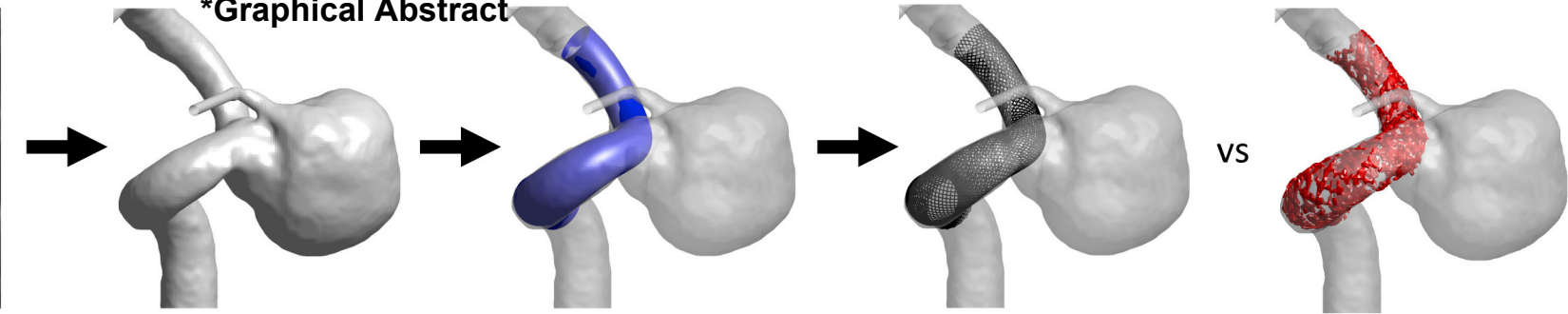


\title{
Geometrical deployment for braided stent
}

\author{
Pierre Bouillot ${ }^{\mathrm{a}, \mathrm{b}}$, Olivier Brina ${ }^{\mathrm{a}}$, Rafik Ouared ${ }^{\mathrm{a}}$, Hasan Yilmaz ${ }^{\mathrm{a}}$, Mohamed Farhat ${ }^{\mathrm{b}}$, Gorislav Erceg ${ }^{\mathrm{a}}$, Karl-Olof \\ Lovblad $^{\mathrm{a}}$, Maria Isabel Vargas ${ }^{\mathrm{a}}$, Zsolt Kulcsar ${ }^{\mathrm{a}}$, Vitor Mendes Pereira ${ }^{\mathrm{a}, \mathrm{c}, \mathrm{d}}$ \\ ${ }^{a}$ Interventional Neuroradiology Unit, Service of Neuroradiology, University Hospital of Geneva \\ ${ }^{b}$ Laboratory for Hydraulic Machines (LMH), Ecole Polytechnique Fédérale de Lausanne (EPFL), Avenue de Cour 33bis, CH-1007 \\ Lausanne, Switzerland \\ ${ }^{c}$ Division of Neuroradiology, Department of Medical Imaging, Toronto Western Hospital, University Health Network, Toronto, Ontario, \\ Canada \\ ${ }^{d}$ Division of Neurosurgery, Department of Surgery, Toronto Western Hospital, University Health Network, Toronto, Ontario, Canada
}

\begin{abstract}
The prediction of flow diverter stent (FDS) implantation for the treatment of intracranial aneurysms (IAs) is being increasingly required for hemodynamic simulations and procedural planning. In this paper, a deployment model was developed based on geometrical properties of braided stents. The proposed mathematical description is first applied on idealized toroidal vessels demonstrating the stent shortening in curved vessels. It is subsequently generalized to patient specific vasculature predicting the position of the filaments along with the length and local porosity of the stent. In parallel, in-vitro and in-vivo FDS deployments were measured by contrast-enhanced cone beam CT (CBCT) in idealized and patient-specific geometries. These measurements showed a very good qualitative and quantitative agreement with the virtual deployments and provided experimental validations of the underlying geometrical assumptions. In particular, they highlighted the importance of the stent radius assessment in the accuracy of the deployment prediction. Thanks to its low computational cost, the proposed model is potentially implementable in clinical practice providing critical information for patient safety and treatment outcome assessment.
\end{abstract}

Keywords: Flow diverter stent, braided stent, virtual deployment, geometrical model, intracranial aneurysm

\section{Introduction}

Flow diverter stents (FDSs) are common endovascular treatment of sidewall intracranial aneurysms (IAs) (Becske et al., 2013; Turjman et al., 2015). Their low porosity aims to moderate and impede the intra-aneurysmal velocities (Pereira et al., 2014a; Larrabide et al., 2015). This flow reduction is known to favor progressive IA thrombosis leading to aneurysm occlusion (Zanaty et al., 2014). Despite of the high treatment efficacy of FDSs (Murthy et al., 2014), it is quite challenging in daily practice to fulfill some of the procedural requirements i.e. IA neck coverage, avoid insufficient stent apposition (ISA) (Heller and Malek, 2011) and side branches coverage (Gascou et al., 2015 ) with proper landing zone. The issue is to select the appropriate device (type, nominal length and diameter) with regard to the specific vessel geometry at the implantation zone. Such issue has highlighted the need of a reliable virtual tool for assisting physicians during stent implantation procedures. Furthermore, computational fluid dynamics (CFD) simulations of IA hemodynamics after FDS implantation will also benefit from realistic virtual stent deployments. Indeed, these simulations have recently shown high potential in predicting the treatment outcome (Pereira et al., 2014b; Mut et al., 2015; Kulcsar et al., 2012; Chong et al., 2013).
During the last decade, considerable efforts have been made to model precisely the stent deployments. Two different approaches were mainly investigated. On one hand, finite element analyses (FEAs) of stent deployment dynamics were performed considering the design and mechanical properties of the device (Kim et al., 2008; Ma et al., 2012, 2013; Bernardini et al., 2012; De Bock et al., 2012). Although very precise and versatile, this approach requires long pre-processing and computational efforts which are difficult to implement in daily clinical practice. On the other hand, phenomenological models based on simplified geometrical or physical constraints were implemented in Janiga et al. (2013); Appanaboyina et al. (2008, 2009); Larrabide et al. (2012); Spranger and Ventikos (2014); Florez Valencia et al. (2004); Bernardini et al. (2012); Mut and Cebral (2012). Their simplifications allowed a strong gain in computational time without significant differences from FEA in the final stent deployment configuration (Bernardini et al., 2012). Nevertheless, quantitative comparisons with real stent implantations were frequently missing.

The widely used braided FDSs have particular design and specific mechanical behavior which can be modeled assuming a limited number of basic geometrical constraints. First, their stable stent braiding and constant wire length suggest a constant inter-wire distance as proposed by Mut 
and Cebral (2012). Second, the quasi-circular cross-section of the stent as usually observed (see Fig 1) is mainly caused by the free rotation of the wires around their stable cross point.

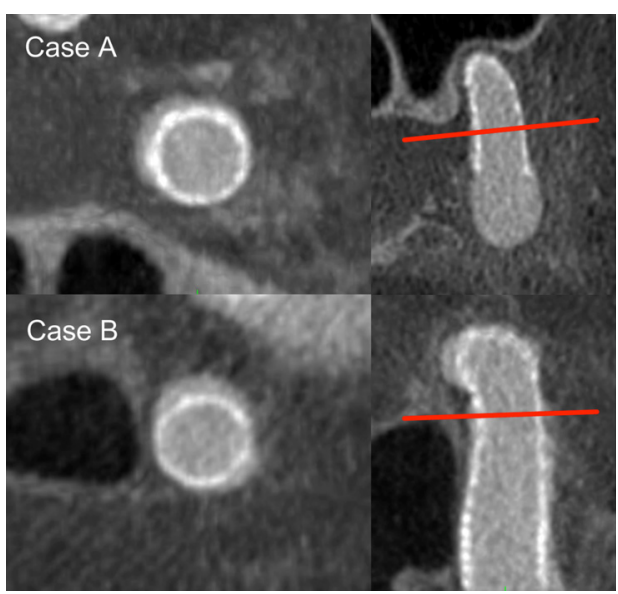

Figure 1: Contrast-enhanced cone beam CT (CBCT) images of two cases acquired immediately after the stent implantation and visualized in multiplanar reconstruction (OsiriX). The left column shows two axial slices positioned orthogonally to the vessel direction as indicated by red lines on the right column. Case A: patient treated with a PED flex 4.5/16 for a right carotid-ophtalmic aneurysm. Case B: patient treated with a PED 4.5/35 for a sub giant aneurysm of the left internal carotid artery siphon. Both cases were not associated with stent delivery issues. These two examples emphasize the circular cross section of the stent restrained in his deployment by the minor radius of an ellipse-shaped artery.

Based on these experimental observations, we have developed a predictive geometrical deployment model specific to braided stents. This model involving only few constraints was entirely defined by a limited number of parameters (number, length and width of wires, inter-wire distance), which were easily assessed on real FDSs. These parameters were enough to predict both the length and local porosity of the deployed stent with a minimal computational cost of about one second on a personal computer. The underlying hypotheses and their consequences were all checked qualitatively and quantitatively on real stent deployment measured by contrast-enhanced cone beam CT $(\mathrm{CBCT})$ in idealized and patient-specific geometries.

\section{Theory}

The deployment model is based on the following geometrical hypotheses, inspired by experimental observations:

- The stent cross section is assumed circular.

- The inter-wire distance $w$ is supposed constant.

The virtual stent is made of $N_{\mathrm{w}}$ cylindrical wires of constant length $l_{\mathrm{w}}$ and diameter $d$ in each braiding direction. Additionally, the intertwining of the wires is neglected. Therefore, the wires cross each other on the tubular stent envelope with local radius $r=(D-d) / 2$ where $D$ is the local diameter of the external envelope of the stent. According to these geometrical constraints the stent unit cell has the diamond-like shape presented in Fig. 2. Its porosity

$$
\varphi(\alpha)=\left[1-\frac{d}{w \sin (2 \alpha)}\right]^{2}
$$

depends only on the local wire angle $\alpha$. It has a symmetric bell-shaped behavior (see Fig. 2) showing a maximum $\varphi_{\max }=(1-d / w)^{2}$ at $\alpha=45^{\circ}$ i.e. for square unit cells.

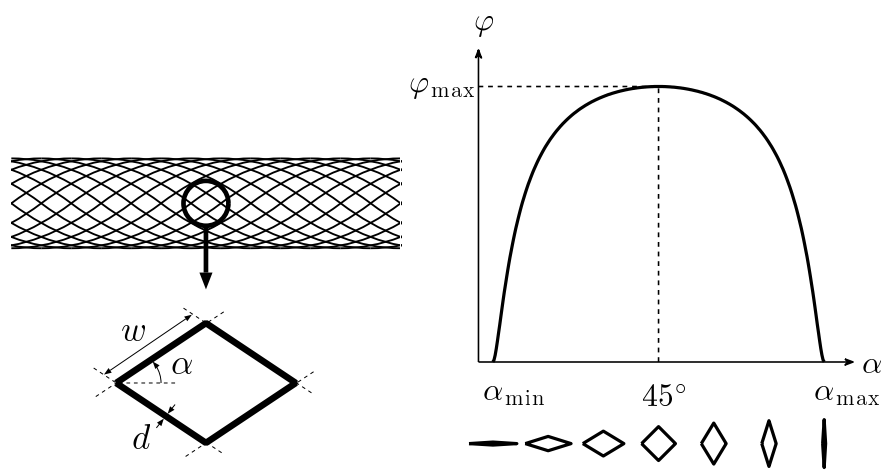

Figure 2: (left) Schematic representation of the diamond-shaped stent unit cell made of wires of diameter $d$ crossing each other with an angle of $2 \alpha$ and separated by a distance $w$. (right) Typical bell-shaped unit cell porosity curve $\varphi(\alpha)$ given in Eq. (1). $\alpha_{\min }=\operatorname{asin}(d / w) / 2$ and $\alpha_{\max }=90^{\circ}-\alpha_{\min }$ are the minimal and maximal wire angles corresponding to closed unit cells.

In the following, the extension of cylindrical stents (Jedwab and Clerc, 1993) to toroidal shapes was first derived and served as a basis for more general tubular deployments subsequently discussed.

\subsection{Geometrical deployment on an open torus}

The envelope of a stent toroidal deployment is described with the angular $(\phi, \theta)$ parametrization

$$
\mathbf{r}(\theta, \phi)=\left(\begin{array}{l}
(R+r \cos \theta) \cos \phi \\
(R+r \cos \theta) \sin \phi \\
r \sin \theta
\end{array}\right) \quad \text { and } s=R \phi
$$

schematized in Fig. 3 where $R$ and $r$ are the major and minor radius of the torus, respectively, and $s$ is the length of the torus centerline. Therefore, the path of each wire is a function $\theta(\phi)$ (or $\theta(s)$, see Fig. 3) which must be computed assuming the above hypotheses i.e. $w$ is constant at every point of the torus. This condition is satisfied by imposing that for a small displacement $\mathrm{d} s$ along the torus centerline the corresponding line element along each wire

$$
\mathrm{d} l=\|\mathrm{d} \mathbf{r}\|=\underbrace{\sqrt{(1+r / R \cos \theta)^{2}+(r \mathrm{~d} \theta / \mathrm{d} s)^{2}}}_{=K} \mathrm{~d} s
$$

is independent of the angular position $\theta$ related to the minor radius. In other words, the winding factor $K$ is 
constant which leads to the relation

$$
\mathrm{d} l=K \mathrm{~d} s= \pm \frac{r}{\sqrt{1-(1+r / R \cos \theta)^{2} / K^{2}}} \mathrm{~d} \theta
$$

numerically integrable to obtain the wire path $\theta(s)$. The two solutions \pm are related to the two braiding directions. With this constraint, the stent length $L=l_{\mathrm{w}} / K$ along the centerline is proportional to the wire length $l_{\mathrm{w}}$. Additionally, the wire angle with respect to the torus centerline (see Fig. 2) becomes

$$
\alpha=\operatorname{asin}\left(r \frac{\mathrm{d} \theta}{\mathrm{d} l}\right)=\operatorname{asin}\left(\sqrt{1-(1+r / R \cos \theta)^{2} / K^{2}}\right) .
$$

It evolves around the minor radius to keep constant $w$.
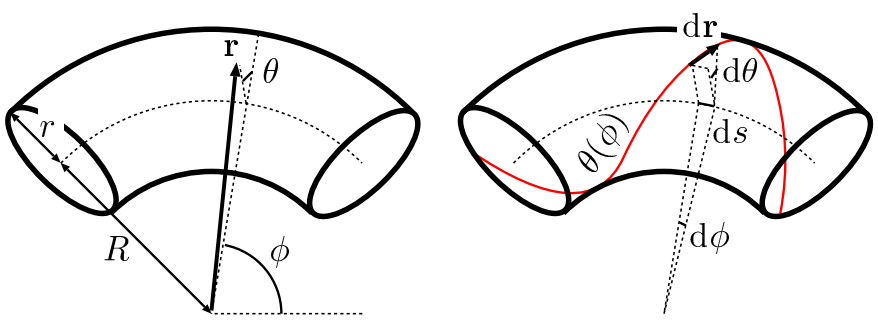

Figure 3: (left) Angular parametrization $\phi$ and $\theta$ of a torus with major $R$ and minor $r$ radius as given in Eq. (2). (right) Representation of a small displacement on the torus dr along a path $\theta(\phi)$ (red line).

The mapping between cylindrical and toroidal deployments being the core of the geometrical model, it is useful to investigate the limit $R / r \rightarrow \infty$ which corresponds to the deployment on a cylindrical envelope of radius $r$. In this case, Eq. (4) has the trivial solution

$$
\theta_{\text {cyl }}\left(s_{\text {cyl }}\right)= \pm \frac{\sqrt{K_{\text {cyl }}^{2}-1}}{r} s_{\text {cyl }}+\theta_{0}
$$

with $K_{\text {cyl }}=\lim _{R / r \rightarrow \infty} K=1 / \cos \alpha_{\text {cyl }}$ and wire angle $\alpha_{\text {cyl }}=\operatorname{asin}\left[\pi r /\left(N_{\mathrm{w}} w\right)\right]$. The constant $\theta_{0}$ depends on the initial position of the wire. This cylindrical solution can be mapped on the toroidal solution (with same minor radius $r$ ) using the transformation

$$
\begin{cases}K_{\mathrm{cyl}} s_{\mathrm{cyl}} & =K s \\ \theta_{\mathrm{cyl}} & =\sqrt{1-1 / K_{\mathrm{cyl}}^{2}} \int_{0}^{\theta} \frac{\mathrm{d} x}{\sqrt{1-(1+r / R \cos x)^{2} / K^{2}}}\end{cases}
$$

derived from Eq. (4) along with the supplementary condition

$$
\frac{1}{2 \pi} \int_{0}^{2 \pi} \frac{\mathrm{d} \theta}{\sqrt{1-(1+r / R \cos \theta)^{2} / K^{2}}}=\frac{1}{\sqrt{1-1 / K_{\mathrm{cyl}}^{2}}}
$$

for $K$. The strict convexity of the function $1 / \sqrt{1-x^{2}}$ imposes the inequality $K>K_{\text {cyl }}$ as can be seen in Fig. 4 where the relation $K_{\text {cyl }} / K$ vs $1 / K_{\text {cyl }}$ is reported for various values of minor/major radius ratio $r / R$. This means that a stent deployed in a torus is always shorter than in a cylinder of the same radius. This stent shortening $L / L_{\text {cyl }}=s / s_{\text {cyl }}=K_{\text {cyl }} / K$ is very small for $r / R \ll 1$ but increases strongly for larger $r / R \lesssim 1$ as indicated in Fig. 4 . Furthermore, the integral in Eq. (8) has a solution only for $K>1+r / R$ limiting the possible braiding configurations especially when $r / R \lesssim 1$.

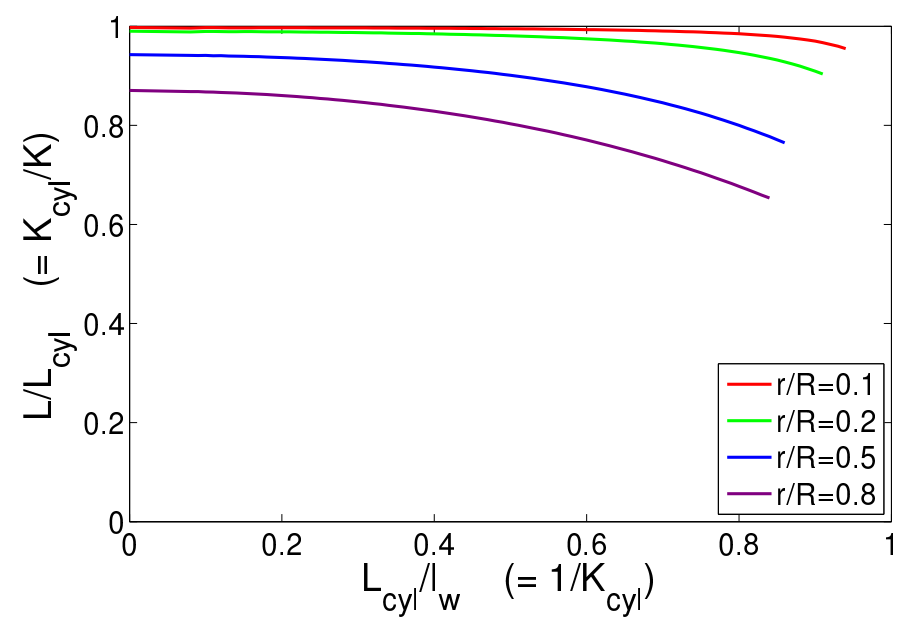

Figure 4: $L / L_{\text {cyl }}$ vs $L_{\text {cyl }} / l_{\mathrm{w}}$ (or $K_{\text {cyl }} / K$ vs $1 / K_{\text {cyl }}$ ) computed from Eq. (8) for various values of minor/major radius ratio $r / R$.

\subsection{Extension to patient-specific vasculature}

The extension of the above results to any tubular stent was obtained by splitting the stent envelope in a sequence of short torus of equal wire length segment with continuously varying radius and curvature. Assuming that the tubular envelope of the stent is inscribed in the patient vasculature, the virtual deployment based on 3D angiograms was decomposed in five steps as shown in Fig. 5. From 3D rotational angiography (3DRA) data, the patient vasculature was first extracted to compute both the centerline and radius of the parent vessel. Once regularized, they served as input to the generation of stent tubular envelope. This envelope was decomposed in short torus to compute Eq. (7) and braid the wires using Matlab (Mathworks, Natick, MA, USA).

1/ vasculature segmentation. The vessel lumen was segmented by applying an interactive watershed analysis (Meyer, 1994) on the gradient of the reconstructed 3DRA DICOM dataset. Two seeding points of the growing regions (inside/outside the parent artery at the proximity of the IA) were manually positioned similar to Higgins and Ojard (1993). The segmented volume was then smoothed with a gaussian filter of $\mathrm{FWHM}=3$ voxels before extracting the boundary surface.

2/ vessel centerline and radius computation. The centerline of the segmented parent vessel was computed using VMTK library (Antiga et al., 2008) (www.vmtk.org) as implemented in Aneufuse software (www.aneurist.org). 


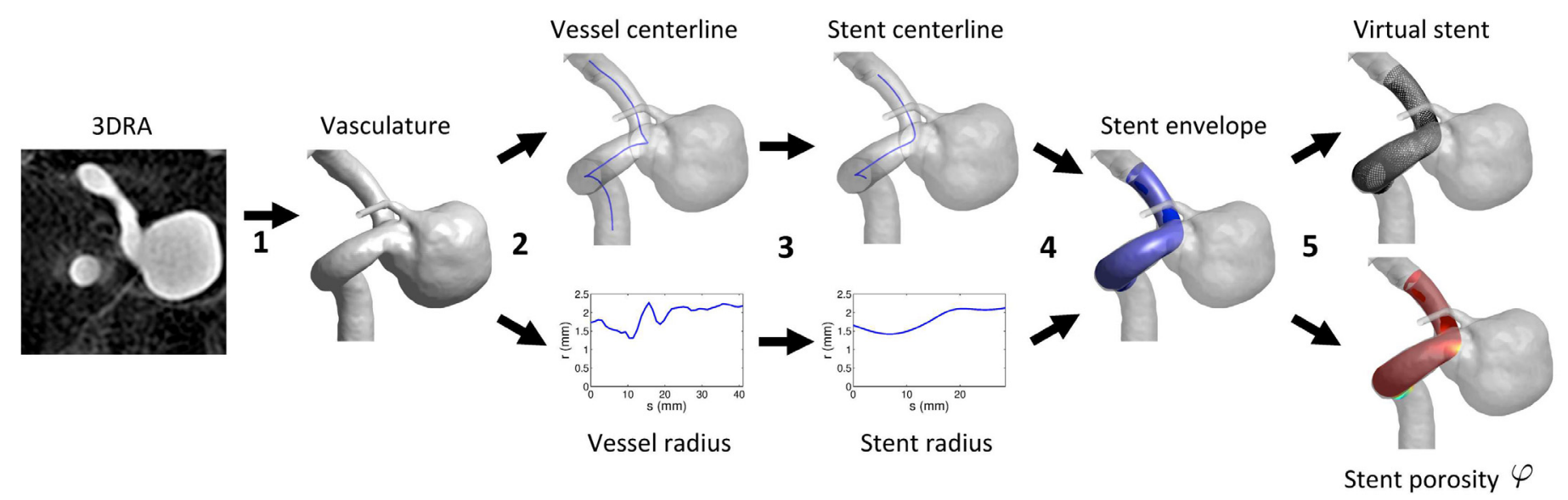

Figure 5: Computational scheme of the virtual stent deployment with the following steps: 1/ vasculature segmentation, 2/ vessel centerline

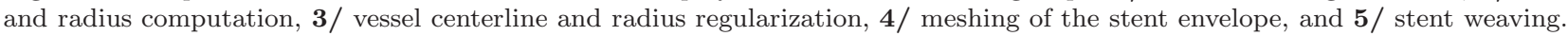

This operation requires the manual selection of two points at the extremities of the parent vessel surface. The vessel radius was subsequently defined as the minimal distance of the vessel boundary from the centerline.

3/ vessel centerline and radius regularization. The stent centerline and radius were approximated from the smoothed vessel values. Additionally, the centerline and radius in the neck segment were both reconstructed in order to minimize the influence of the IA and neck size. For this purpose, two points delineating the proximal/distal extremity of the neck were manually selected on the centerline. The centerline/radius were then regularized using smoothing splines after removing partially/totally the neck segment and the radius larger than the maximal stent radius $\alpha_{\max }$. Note that the wire radius $d / 2$ was first subtracted from the vessel radius to take into account the finite width of the stent wires. The local radius of curvature $R$ along with the tangent $\hat{\mathbf{T}}$ and normal $\hat{\mathbf{N}}$ unit vectors were subsequently computed from the spline expressions (see Fig. 6.a) and parametrized with the length $s$ of the regularized centerline. (a)

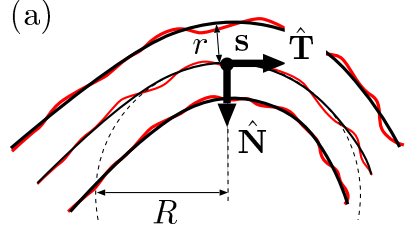

(b)

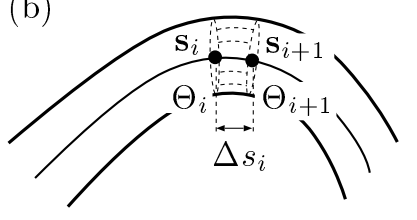

Figure 6: (a) Centerline and radius of the parent vessel before (red lines) and after (black lines) regularization. $\hat{\mathbf{T}}$ and $\hat{\mathbf{N}}$ are the tangent and normal unit vectors at the position $\mathbf{s}$ along the regularized centerline. $R$ and $r$ are the regularized radius of curvature and radius of the vessel. (b) $i^{\text {nd }}$ toroidal element of the stent envelope decomposition between $\mathbf{s}_{i}$ and $\mathbf{s}_{i+1} . \Theta_{i}=\left\{\theta_{i, j}\right\}_{j}$ and $\Theta_{i+1}=\left\{\theta_{i+1, j}\right\}_{j}$ are the corresponding minor axis angular decomposition at both ends of this toroidal element of length $\Delta s_{i}$.

4/ meshing of the stent envelope. In order to guarantee the spatial uniformity and continuity of the wires on the stent envelope, the latter was meshed in $M \times N$ nodes $\mathbf{r}_{i=1 . . M, j=1 . . N}^{\mathrm{m}}$ which were parametrized by the wire length and polar angle $\left(l_{\mathrm{cyl}, i}, \theta_{\mathrm{cyl}, j}\right)$ of a cylindrical deployment (see Fig. 7). In this way, the cylindrical solution Eq. (6) was easily mapped on the patient vasculature. For this purpose, the stent envelope was decomposed in $M$ short torus with constant wire length segment centered on the regularized centerline at $\mathbf{s}_{i=1 . . M}=\mathbf{s}\left(s_{i=1 . . M}\right)$ (see Fig. 6.b). Assuming weakly varying stent radius and curvature, each short torus was considered independent from each other to mesh the stent envelope according to the theory developed in Sec. 2.1. The length $\Delta s_{i}=s_{i+1}-s_{i}$ of each toroidal segment (between $\mathbf{s}_{i}$ and $\mathbf{s}_{i+1}$ ) was selected to induce a constant wire length $\Delta l$ as in Eq. (4). Furthermore, the angular subdivision $\Theta_{i}=\left\{\theta_{i, j}\right\}_{j=1 \ldots N}$ was adapted locally to satisfy the mapping of Eq. (7) from a uniform angle discretization $\theta_{\mathrm{cyl}, j}=2 \pi(j-1) / N$ on the cylinder. In this way, the generated mesh $\mathbf{r}_{i, j}^{\mathrm{m}}$ was parametrized by the discretized wire length $l_{\mathrm{cyl}, i} \stackrel{\mathrm{r}}{=}$ $(i-1) \Delta l$ and polar angle $\theta_{\mathrm{cyl}, j}$ of a cylindrical deployment. More precisely, the spatial location of the $M \times N$ nodes of the mesh $\mathbf{r}_{i, j}^{\mathrm{m}}$ was iteratively computed as presented in Algorithm 1.

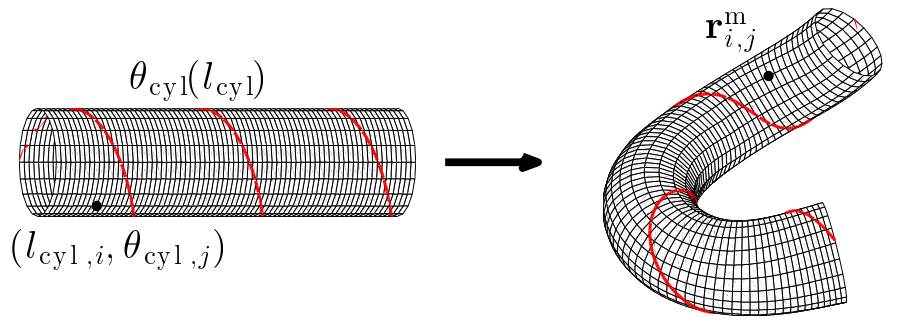

Figure 7: Wire path $\theta_{\text {cyl }}\left(l_{\text {cyl }}\right)$ of a cylindrical deployment (Eq. (6)) mapped on the patient specific stent envelope (red lines) through the parametrization $\left(l_{\mathrm{cyl}, i}, \theta_{\mathrm{cyl}, j}\right) \rightarrow \mathbf{r}_{i, j}^{\mathrm{m}}$ of the meshed stent envelope.

$\mathbf{5}$ / stent weaving. First, the distal end of the stent was manually selected on the regularized centerline. Then the 
Input: At the initial $\left(s_{1}=0\right)$ centerline position $\mathbf{s}_{1}$ : stent and curvature radius $r_{1}$ and $R_{1}$, tangent and normal vectors $\hat{\mathbf{T}}_{1}$ and $\hat{\mathbf{N}}_{1}$, orthonormal coordinate system $\mathbf{v}_{1,1}=\hat{\mathbf{T}}_{1}$, $\mathbf{v}_{2,1}, \mathbf{v}_{3,1}$

for $i=1 . . M$ do

Extract $K_{i}$ from Eq. (8);

Build the angular discretization

$\Theta_{i}=\left\{\theta_{i, j}\right\}_{j=1 . . N}$ at $\mathbf{s}_{i}$ according to Eq. (7) and the direction of curvature $\hat{\mathbf{N}}_{i}$;

Locate the corresponding mesh nodes $\mathbf{r}_{i, j}^{\mathrm{m}}=\mathbf{s}_{i}+r_{i}\left(\mathbf{v}_{2, i} \cos \theta_{i, j}+\mathbf{v}_{3, i} \sin \theta_{i, j}\right) ;$

Compute the length $\Delta s_{i}=K_{i} \Delta l$ of the torus segment;

Move to the next centerline position $\mathbf{s}_{i+1}=\mathbf{s}\left(s_{i+1}\right)$ with $s_{i+1}=s_{i}+\Delta s_{i}$;

Determine the corresponding stent and curvature radius $r_{i+1}$ and $R_{i+1}$, tangent and normal vectors $\hat{\mathbf{T}}_{i+1}$ and $\hat{\mathbf{N}}_{i+1}$;

Apply the rotation occurred between $\hat{\mathbf{T}}_{i}$ and $\hat{\mathbf{T}}_{i+1}$ on the cartesian coordinate system $\mathbf{v}_{\{1,2,3\}, i}$ to get $\mathbf{v}_{\{1,2,3\}, i+1}$;

end

Algorithm 1: Meshing of the stent envelope.

cylindrical solution Eq. (6) of the $k_{ \pm}=0 . . N_{\mathrm{w}}-1$ uniformly distributed wire paths i.e. $\theta_{\text {cyl }}^{k_{ \pm}}\left(l_{\text {cyl }}\right)= \pm \pi /\left(w N_{\mathrm{w}}\right) l_{\text {cyl }}+$ $2 \pi k_{ \pm} / N_{\mathrm{w}}+\theta_{0}$ starting from the selected position $l_{\mathrm{cyl}}=l_{\text {init }}$ and finishing at $l_{\mathrm{cyl}}=l_{\text {init }}+l_{\mathrm{w}}$ was interpolated on the parametric mesh $\left(l_{\mathrm{cyl}, i}, \theta_{\mathrm{cyl}, j}\right) \rightarrow \mathbf{r}_{i, j}^{\mathrm{m}}$ as shown in Fig. 7 .

\subsection{Stent parameters}

In our geometrical model, each stent is entirely described by its parameters $d, w, N_{\mathrm{w}}$ and $l_{\mathrm{w}}$. Although the model applies to any braided stent, in the present work we focussed on Pipeline embolization devices (PEDs) (Covidien, Irvine, USA) which are commonly used at Geneva university hospitals (HUG). PEDs are made of $N_{\mathrm{w}}=24$ wires of diameter $d=33 \mu \mathrm{m}$ (Bouillot et al., 2014a) in each braiding direction. Concerning the inter-wire distance $w$, it was assessed for all the available PEDs by fitting the geometrical model of a cylindrical deployment derived from Eq. (6) on the manufacturer data in Fig. 8. Table 1 summarizes the obtained values along with the nominal wire angle $\alpha_{\text {nom }}$ i.e. the wire angle of the stent deployed in its nominal diameter. This quantity is important since it allows to compute the wire length $l_{\mathrm{w}}$ from Eq. (6) and the two PED identifications numbers i.e. the nominal diameter/length. The maximum porosity $\varphi_{\max }$ at $\alpha=45^{\circ}$ and the corresponding tube diameter $D_{\max }$ were also derived from Eqs. (1) and (6). These values are in total agreement with the measured porosities given in Shapiro et al. (2014).
Moreover, due to the fixed number of wires in PEDs, their inter-wire distance $w$ increases with their nominal diameter $D_{\text {nom }}$ insuring an almost constant nominal wire angle $\alpha_{\text {nom }}$. Consequently, the maximal porosity of PEDs $\phi_{\max }$ increases with its nominal diameter from 0.67 to 0.82 .

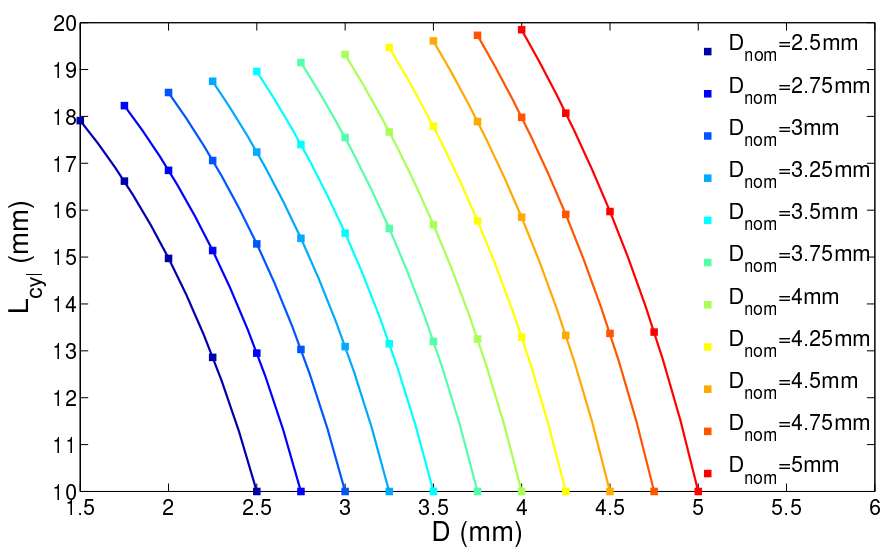

Figure 8: Length $L_{\mathrm{cyl}}$ of PEDs deployed in a tube of diameter $D$ with nominal length $L_{\text {nom }}=10 \mathrm{~mm}$ and various nominal diameters $D_{\text {nom }}$. The geometrical model derived from Eq. (6) (full lines) is fitted on the manufacturer data (squares) with a unique free parameters i.e. the inter-wire distance $w$ given in Table 1 .

\section{Measurements}

The accuracy of the deployment model was tested against real FDS implantations measured with CBCT (vasoCT - Philips Healthcare, Best, the Netherlands). To this aim, the raw acquisition volume of the CBCT was reconstructed on a cubic mesh centered on the region of interest with isotropic voxels of width $0.069 \mathrm{~mm}$ or $0.1345 \mathrm{~mm}$ depending on the size of the volume of interest. The radioopaque wires of the stent ( 1 wire over 4 for PEDs) were subsequently segmented using a manual thresholding and aligned on the virtual deployment. Therefore the properties of the real stent were locally comparable with those of the virtual stent.

In order to verify the two main hypotheses of the deployment model (circular stent cross section and constant inter-wire distance $w$ ) and its predictions, the shape of the stent envelope and the braiding properties were both analyzed on real FDSs.

\subsection{Stent cross section}

The stent envelope was measured assuming an elliptic cross section characterized by its major and minor radius ( $a$ and $b$ ) and eccentricity $e$. For this purpose, orthogonal slices along the regularized centerline were first extracted from the CBCT data (see Fig. 9.a). Then the elliptic cross section maximizing the CBCT intensity at its boundary was computed on each slice using a Nelder-Mead simplex (Lagarias et al., 1998) optimization algorithm implemented in Matlab (Mathworks, Natick, MA, USA). These measurements were restricted to the central part of the 


\begin{tabular}{lccccccccccc}
\hline$D_{\text {nom }}(\mathrm{mm})$ & 2.5 & 2.75 & 3 & 3.25 & 3.5 & 3.75 & 4 & 4.25 & 4.5 & 4.75 & 5 \\
\hline$w(\mathrm{~mm})$ & 0.1836 & 0.1995 & 0.2155 & 0.2315 & 0.2476 & 0.2636 & 0.2798 & 0.2959 & 0.3121 & 0.3283 & 0.3445 \\
$\alpha_{\text {nom }}\left(^{\circ}\right)$ & 61.6 & 63.1 & 64.3 & 65.5 & 66.4 & 67.3 & 68.1 & 68.8 & 69.5 & 70.1 & 70.7 \\
$\varphi_{\max }$ & 0.67 & 0.70 & 0.72 & 0.74 & 0.75 & 0.77 & 0.78 & 0.79 & 0.80 & 0.81 & 0.82 \\
$D_{\max }(\mathrm{mm})$ & 2.02 & 2.19 & 2.36 & 2.53 & 2.71 & 2.88 & 3.06 & 3.23 & 3.41 & 3.58 & 3.76 \\
\hline
\end{tabular}

Table 1: Inter-wire distance $w$, nominal wire angle $\alpha_{\text {nom }}$ and maximum porosity $\varphi_{\max }$ (in a tube of diameter $D_{\max }$ ) for PEDs with various nominal diameters $D_{\text {nom. }}$. These values are extracted from the fit of Eq. (6) on the manufacturer data shown in Fig. 8.

stent. Indeed, the partial visualization of the opaque wires on orthogonal slices located at less than $\sim 1 \mathrm{~mm}$ of both ends prevented a reliable cross section measurement.

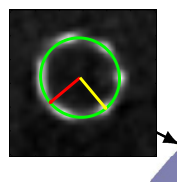

(a)

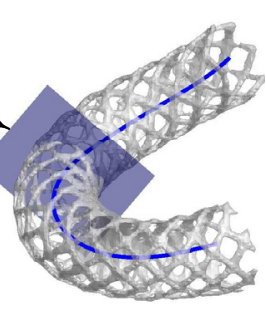

(b)

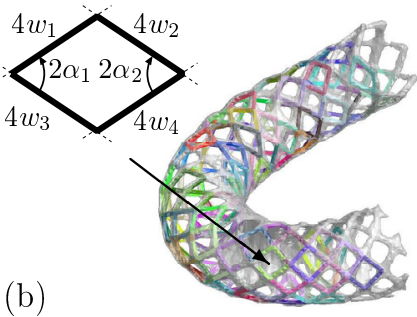

Figure 9: (a) CBCT slice (transparent blue plane) orthogonal to the virtual stent centerline (blue line) with the optimal elliptic stent cross section (green) and its major/minor radius (yellow/red arrow) upon which the CBCT intensity is maximized. (b) Radio-opaque wires (1 wire over 4 for PEDs) acquired from CBCT measurements. The colored diamonds are the measured unit cells where each vortex maximizes the $\mathrm{CBCT}$ value along the two crossing wire directions. The inset shows the geometrical parameters measured on these CBCT unit cells: the 2 opposite wire angles $\alpha_{1,2}$ and the 4 inter-opaquewire distances $4 w_{1,2,3,4}$

\subsection{Wire angle and inter-wire distance}

The measurement of wire angles $\alpha$ and inter-wire distances $w$ required the implementation of a semi-automatic tool. The four nodes of each measured unit cell were first positioned manually on the CBCT segmented surface (see Fig. 9.b). These node locations were then optimized such that the CBCT intensity was maximized along the two directions of the crossing wires using the simplex algorithm previously cited. The two opposite angles oriented towards the centerline direction $\left(2 \alpha_{1,2}\right)$ were then computed along with the four inter-node distances $\left(4 w_{1,2,3,4}\right.$ for PEDs) and unit cell center.

\section{Experiments}

Four virtual deployments were compared to CBCT acquisitions of real in-vivo and in-vitro FDS implantations in various idealized and patient specific configurations.

\subsection{In-vitro deployments}

FDSs were first implanted in silicone models (Elastrat SA) manufactured with the lost wax technique (Wetzel et al., 2005) from 3D printings of the following idealized and patient specific geometries:
- Torus with minor and major radius, $r=1.6 \mathrm{~mm}$ and $R=5 \mathrm{~mm}$, respectively, implanted with a PED 3.75/16 to test the toroidal deployment in Sec. 2.1.

- Cone of length $40 \mathrm{~mm}$ with radius increasing from 1 to $2 \mathrm{~mm}$, implanted with a PED $3.5 / 25$ to verify the weakly varying radius hypothesis in Sec. 2.2 .

- Vasculature of a patient carrying a left carotid ophthalmic IA segmented from 3DRA data and implanted with a PED 4/16 to test the virtual deployment in patient specific geometry.

In order to avoid vessel shape modifications during the stent implantation, the silicone models were molded in large blocks with rigid vessels. Each model underwent a 3DRA to evaluate its geometrical accuracy with regard to the original 3D data. Typical deviations of the order of $100 \mu \mathrm{m}$ were detected as previously described in Bouillot et al. (2014b).

PEDs were implanted by confirmed neuroradiologists according to the clinical requirements after having inserted the silicone model in a circulating set-up filled with a mixture of glycerin $(59.1 \%)$ and water $(40.9 \%)$. This circulating fluid was heated at $37^{\circ} \mathrm{C}$ for ensuring the correct deployment of thermal shape-memory PED. The fluid matched the refraction index $n_{\mathrm{f}}=1.409$ of the silicone thus preventing optical deformations at the vessel wall for direct optical imaging of actual FDS implantations. Pictures of the toroidal and conical deployments were performed for additional comparison.

Contrarily to in-vivo measurements, the in-vitro CBCT acquisitions are not affected by the surrounding tissues and bones. These measurements thus guaranteed good quality data for analyzing the stent envelope and braiding properties.

Since the geometrical model is very sensitive to the actual stent radius, the prediction of the virtual deployments (cone and torus) were based on the real stent dimensions assessed with CBCT. For instance, the toroidal deployment was performed with minor radius $r=1.52 \mathrm{~mm}$ corresponding to the measured real stent radius (see inset of Fig. 12) which is slightly lower than the silicone model dimension $(r=1.6 \mathrm{~mm})$.

\subsection{In-vivo deployment}

In order to test the virtual deployment in clinical situation, a patient carrying a right internal carotid artery 
IA (ophthalmic segment) treated with a PED 4.75/16 was also selected. The 3DRA data before stenting served as an input of the virtual deployment. The clinical part of this study was made under the Ethical Comittee authorization (12-179) for the acquisition and the processing of patient data.

\section{Results}

The comparisons between real and predicted stent implantations are splitted in two parts. First deployments in idealized geometries (torus and cone) are presented to check the assumptions of the theoretical model. Then the virtual deployment abilities in more complex patient specific vasculatures are assessed in-vitro and in-vivo.

\subsection{Deployment in idealized models}

Qualitative comparisons of the virtual vs real deployments were provided by the superimposition of the predicted wires location on both rescaled pictures and segmented CBCTs of the real deployments in Figs. 10 and 11. These figures show very good visual agreement in both torus and cone regarding the wire repartition and orientation which were quantified by measuring the inter-wire distances $w$ and angles $\alpha$. For these purposes, a selection of connected real stent unit cells was analyzed on the basis of CBCT according to Sec. 3.2. These measurements covered fully the minor axis angle $\theta$ of the torus and the diameter $D$ of the cone.

(a)

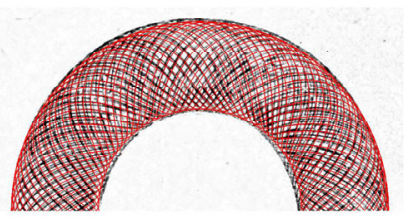

(b)

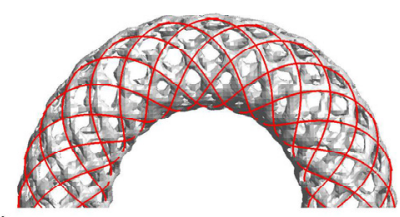

Figure 10: Toroidal deployment of a PED 3.75/16. The virtual deployment predicted by the geometrical model (red lines) is superimposed on a picture of the real stent (a) and the segmented CBCT measurement in (b) where only the radio-opaque wires are visible (1 wire over 4 ).

(a)
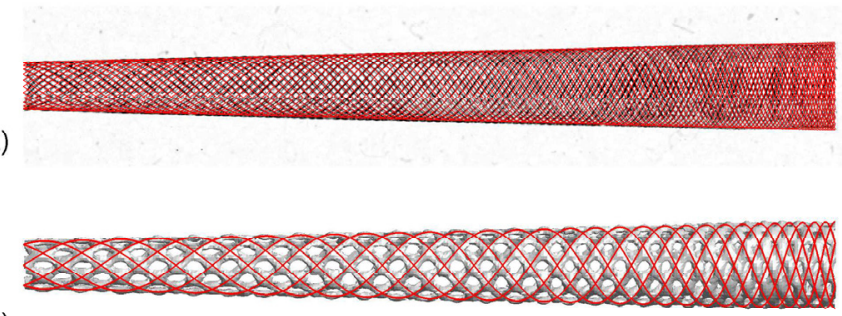

(b)

Figure 11: Conical deployment of a PED 3.5/25. The virtual deployment predicted by the geometrical model (red lines) is superimposed on a picture of the real stent (a) and the segmented CBCT measurement in (b) where only the radio-opaque wires are visible (1 wire over 4$)$.
The average and standard deviation of the measured inter-wire distances $w \pm \sigma_{w}$ were $0.246 \pm 0.018 \mathrm{~mm}$ and $0.235 \pm 0.015 \mathrm{~mm}$ for the toroidal and conical deployments, respectively. These values are very close to the expected $0.2636 \mathrm{~mm}$ and $0.2476 \mathrm{~mm}$ for PED $3.75 / 16$ and PED 3.5/25 (see Table 1), respectively. Furthermore, the small experimental variations of $60-70 \mu \mathrm{m}$ on the measured CBCT unit cells i.e. $\sigma_{w} / w \approx 6-7 \%$ is below the instrument resolution. This confirmed the relevance of the constant $w$ assumption in the deployment model and the good agreement of the wire repartition found in Figs. 10 and 11.

Concerning the measured wire angle $\alpha$, its behavior with respect to the the minor axis angle $\theta$ of the torus and along the diameter $D$ of the cone was compared with the theoretical prediction Eq. (5) in Figs. 12 and 13.

Torus. The predicted cosine like behavior of the theoretical $\alpha(\theta)$ related to the closing/opening of the unit cells outwards/inwards the torus was well reproduced by the measured angles. Although the theoretical model was free of tunable parameter, its results matched well with the experiments both in shape and amplitude. This confirmed the theoretical bases of the geometrical model and the wire orientation agreement in Fig. 10.

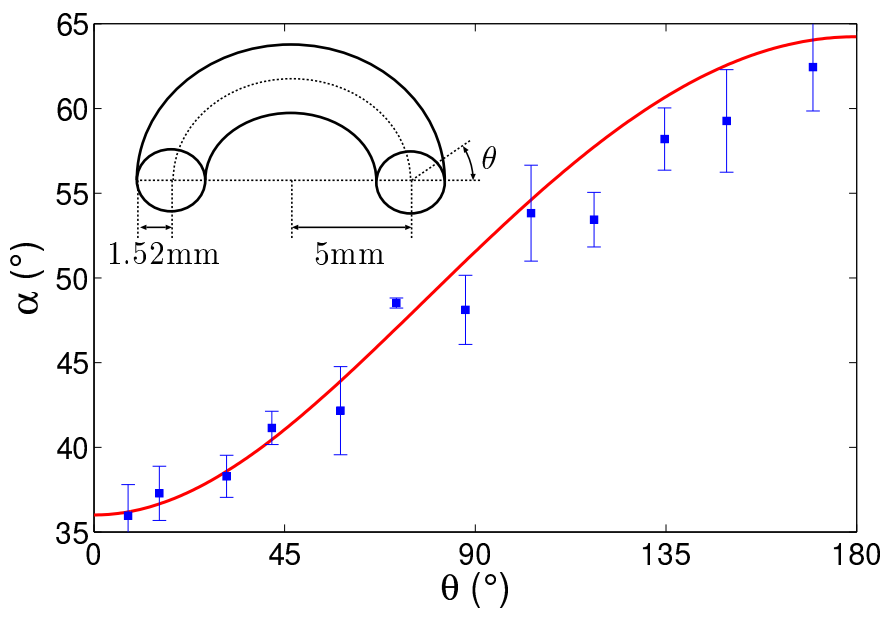

Figure 12: Wire angle $\alpha$ plotted vs the minor axis angle $\theta$ of the toroidal deployment shown in Fig. 10 and represented in the inset. The blue squares represent the measurements based on CBCT acquisition and the red line is the theoretical prediction Eq. (5) with $\mathrm{R}^{2}=0.94$. The error bars show one standard deviation computed from the two opposite angles (see Fig. 9.b) measured in two unit cells.

Cone. The opening of the unit cells measured with increasing implantation diameter along the cone was in complete agreement with the theoretical model $\alpha(D)$ based on a slowly varying radius approximation. Furthermore, the length of both virtual and real stent matched perfectly (Fig. 11). Again, these results confirmed the assumptions of the deployment model and the wire orientation agreement in Fig. 11. It is worth noting that the implanted PED did not expand more than its nominal diameter $\approx 3.5 \mathrm{~mm}$. 


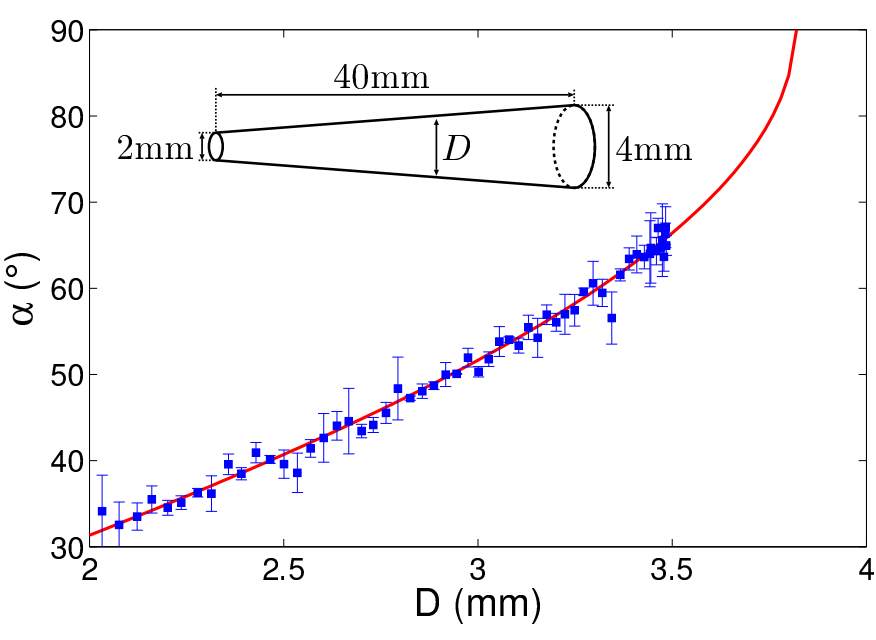

Figure 13: Wire angle $\alpha$ plotted vs the diameter $D$ of the conical deployment shown in Fig. 11 and represented in the inset. The blue squares represent the measurements based on a CBCT acquisition and the red line is the theoretical prediction Eq. (5) with $\mathrm{R}^{2}=0.99$. The error bars show one standard deviation computed from the two opposite angles (see Fig. 9.b) measured in one unit cell.

\subsection{Patient specific deployment}

Real and virtual stents implanted in-vitro and in-vivo in patient vasculatures are compared in Figs. 14.a-b.1. These figures show the high accuracy of the geometrical model prediction performed without free stent parameters. First, the stent length $L$ was precisely predicted for both the in-vitro $(20.7 \mathrm{~mm})$ and in-vivo $(28.5 \mathrm{~mm})$ implantations despite of the complex shape of the vasculatures (length difference $<1 \mathrm{~mm}$ between the real and virtual stents). This result was found closely related to the assumed virtual stent cross section which matched well the real stent. As shown in Figs. 14.a-b.3 and summarized in Table 2 for the two in-vitro and in-vivo cases, the shape and dimensions of the real and virtual cross sections were very close along the stent centerline. On the one hand, the circular cross section assumed on virtual stents matched with the low eccentricity of the real stent $(e \approx 0.2-0.3$ in average corresponding to minor/major radius differences of only few percents). On the other hand, the vessel radius measured on the pre-stent 3DRA acquisition gave a good approximation of the implanted stent radius, except at the proximal end of the in-vitro implantation where the stent did not deploy fully. As expected, this radius increased toward the proximal end of the stent.

It was also possible to estimate the local stent porosity $\varphi$ (Figs. 14.a-b.2) directly from Eqs. (1) and (5). Due to the bell-shaped function $\varphi(\alpha)$ in Fig. 2, the porosity was relatively constant over the most part of the virtual stent envelope. However, the area with lower porosity value correlated well with higher metal strut density in segmented CBCT of the real stent.

Alternatively, the better quality of the in-vitro CBCT acquisition (compared to in-vivo) allowed to measure both the inter-wire distances $w$ and angles $\alpha$ as in idealized

\begin{tabular}{lcccccc}
\hline & $\bar{r}_{\text {vir }}$ & $\bar{a}_{\text {mes }}$ & $\bar{b}_{\text {mes }}$ & $\bar{r}_{\text {mes }}$ & $\Delta \bar{r}_{\text {mes }} / \bar{r}_{\text {mes }}$ & $\bar{e}_{\text {mes }}$ \\
\hline in-vitro & 1.69 & 1.69 & 1.60 & 1.65 & 0.05 & 0.30 \\
in-vivo & 1.78 & 1.80 & 1.76 & 1.78 & 0.03 & 0.21 \\
\hline
\end{tabular}

Table 2: Virtual stent radius $r_{\text {vir }}$ in $(\mathrm{mm})$, real stent cross section parameters (minor, major and mean radius $a_{\text {mes }}, b_{\text {mes }}$ and $r_{\text {mes }}$ in $(\mathrm{mm})$, radius difference $\Delta r_{\mathrm{mes}}=a_{\mathrm{mes}}-b_{\mathrm{mes}}$ and associated eccentricity $\left.e_{\text {mes }}=\sqrt{1-\left(b_{\text {mes }} / a_{\text {mes }}\right)^{2}}\right)$ from Fig. 14.a-b.3 averaged along the stent centerline.

geometries. For this purpose, 104 uniformly distributed CBCT unit cells covering fully the FDS surface (see the colored unit cells in Fig. 9.b) were analyzed. Only unit cells located in strong vessel curvature could not be assessed since the opaque wires merged in the 3D reconstruction of CBCT acquisitions.

The measured $w \pm \sigma_{w}$ was $0.272 \pm 0.015 \mathrm{~mm}$ in agreement with both the virtual deployment $(0.280 \pm 0.006 \mathrm{~mm})$ and the nominal value for the implanted PED 4/14 $(0.2798 \mathrm{~mm}$, see Table 1$)$. These results confirmed again the constant inter-wire assumption which was respected in both the real and virtual stents. Consequently the total length $l_{\mathrm{w}}$ of the virtual stent wires $37.5 \pm 0.1 \mathrm{~mm}$ was very close to the expected value of $37.56 \mathrm{~mm}$ for PED 4/14.

Fig. 15 compares the measured wire angles $\alpha_{\text {mes }}$ with the virtual value $\alpha_{\text {vir }}$. For consistent virtual vs real stent comparison, the center of each measured CBCT unit cell was located on the virtual stent envelope. One can see in this figure that the virtual and real wire angles (blue squares) reasonably correlated $\left(\mathrm{R}^{2}=0.49\right)$ and were located around the ideal case $\alpha_{\text {mes }}=\alpha_{\text {vir }}$. However in average, $\alpha_{\text {vir }}$ slightly overestimated $\alpha_{\text {mes }}$ by $2.6^{\circ} \pm 4.9^{\circ}$. Moreover, the outliers located mainly at the proximal part of the virtual stent, which had a slightly overestimated radius compared to the real stent, were reduced $\left(\mathrm{R}^{2}=0.75\right)$ if the real stent radius was considered instead in the virtual deployment (green circle). In this case, the points were recentred in the vicinity of the identity curve $\alpha_{\text {mes }}=\alpha_{\text {vir }}$ decreasing the virtual angle overestimation to $0.7^{\circ} \pm 3.9^{\circ}$. This result emphasizes the high sensitivity of the wire angles with the stent radius and therefore the importance of the stent radius estimation in the virtual deploymenst methodology (Fig. 5).

\section{Discussion}

In this manuscript, we have demonstrated the ability of the mathematical model to accurately predict the implantation of braided stents in various configurations. This geometrical model is fully defined by a limited number of hypotheses (i.e. constant inter-wire distance and circular stent cross section) and parameters (i.e. wire number, width and length, and inter-wire distance), which were either deduced from manufacturer data or experimentally assessed. Therefore, the virtual deployment predicting the wire locations together with the stent length and porosity 


\section{Silicone Model}

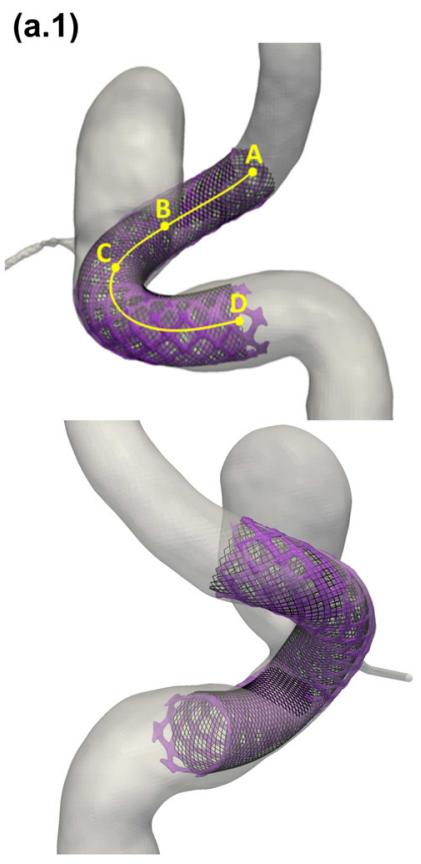

(a.2)
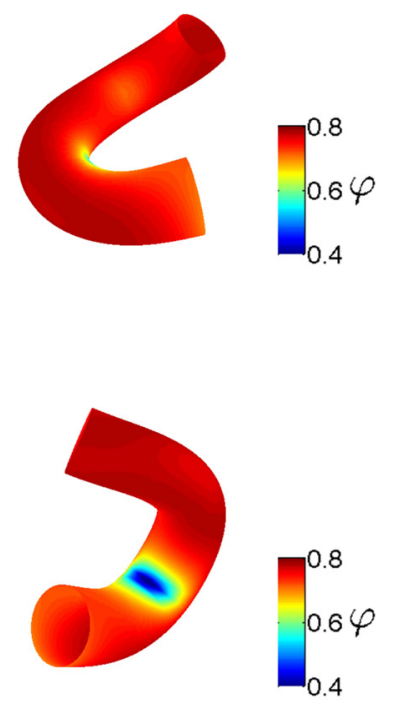

Patient Case

(b.1)

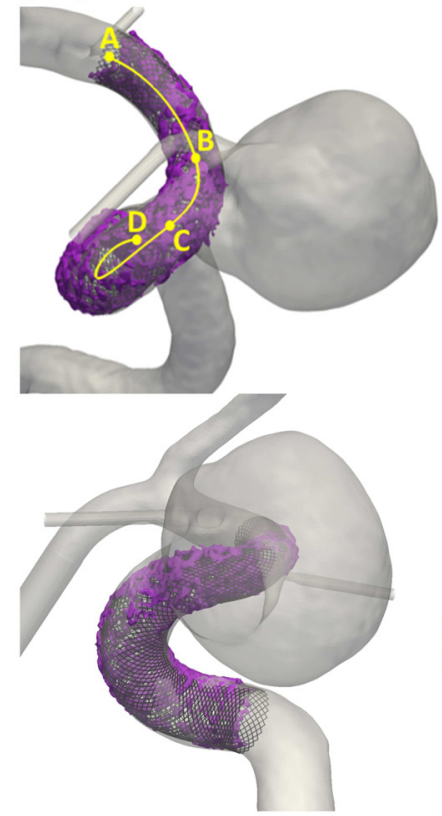

(b.2)
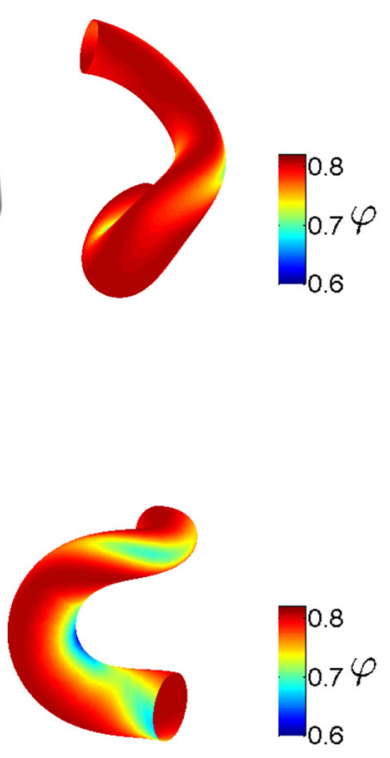

(a.3)

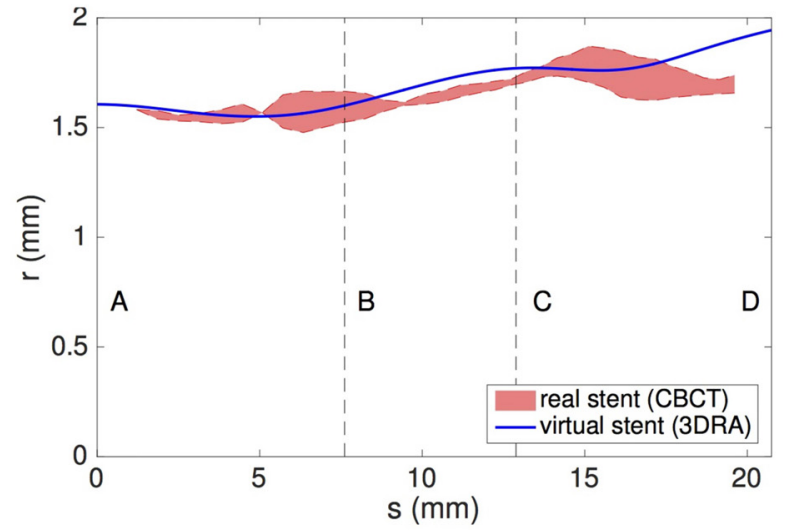

(b.3)

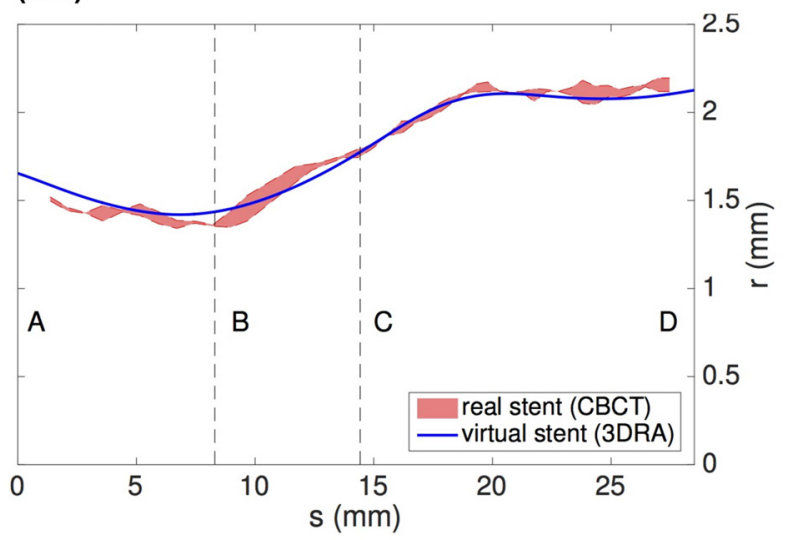

Figure 14: In-vitro (column (a)) and in-vivo (column (b)) stent deployment in patient specific geometries. (a.1 and b.1) show a comparison between the virtual and real stents visualized on Paraview (www.paraview.org) in two different orientations. The virtual stent represented by black lines is superimposed on the radio-opaque wires of the real stent segmented from CBCT acquisition (purple surfaces). The semitransparent surface is the pre-stent vascular geometry segmented from 3DRA. (a.2 and b.2) show the local porosity of the virtual stent in the same orientations as (a.1 and b.1). (a.3 and b.3) show the radius $r$ of the stent vs the position $s$ along the stent centerline (the origin is taken at the distal end). The blue line represents the radius of the virtual stent $r_{\mathrm{vir}}$ based on 3DRA pre-stent acquisition while the red area shows the minor-major radius range $\left[b_{\mathrm{mes}}, a_{\mathrm{mes}}\right]$ of the real stent cross section based on CBCT post-stent acquisition (see Sec. 3.1 and Fig. 9.a). The labels (A, B, C, D) correspond to the proximal and distal ends of the stents as well as the neck location marked along the stent centerline in (a.1 and b.1). 


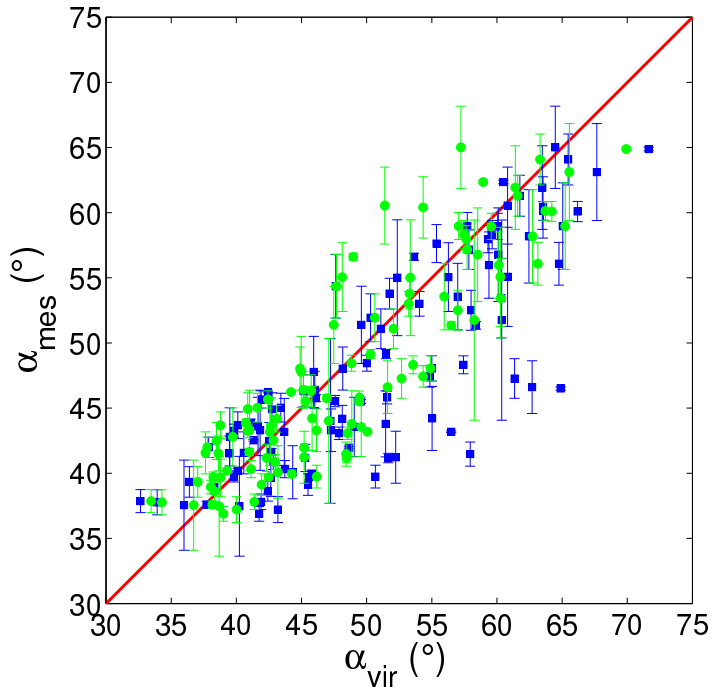

Figure 15: Comparison between real and predicted wire angles of a PED 4/14 deployed in the in-vitro model shown in Fig. 14.a. 104 randomly measured angles $\alpha_{\text {mes }}$ (see Fig. 9.b) were compared to the predicted angles $\alpha_{\text {vir }}$ of the virtual deployment at the same stent envelope locations. The blue boxes represent the angles of the virtual deployment based on the 3DRA vasculature while the green circles are those of the corrected deployment based on the real stent radius measured by CBCT. The red line represents the ideal case $\alpha_{\text {mes }}=$ $\alpha_{\text {vir }} . \mathrm{R}^{2}=0.49$ for the original 3DRA based virtual deployment and $\mathrm{R}^{2}=0.75$ for the corrected CBCT based virtual deployment. The error bars represent one standard deviation.

was performed without any free parameters. As to the hypotheses, both of the inter-wire distance and the circular cross section have been directly verified experimentally. However, the constant winding factor in toroidal deployment was indirectly confirmed by the good agreement between both the predicted and measured wire angles. The effect of continuous radius variations on the virtual deployment was found negligible in the cone. The application of the deployment model to patient specific geometries, was therefore facilitated by the toroidal decomposition of the implanted vessel, hence mimicking accurately the real stent behavior.

Fundamental consequences of the underlying theoretical models were also highlighted. In particular, it has been demonstrated mathematically and under constant interwire distance hypothesis, how braided stent shortening in toroidal deployments was correlated with the vessel curvature.

\subsection{Technical discussion}

Unlike most of the previous phenomenological models (Janiga et al., 2013; Appanaboyina et al., 2008, 2009; Larrabide et al., 2012; Spranger and Ventikos, 2014; Florez Valencia et al., 2004; Bernardini et al., 2012; Mut and Cebral, 2012) where the stent envelope followed closely the vessel walls, a tubular envelope inscribed in the vessel lumen was assumed in agreement with the in-vivo observations presented in Fig. 1 and the cross-sections measured from real deployments of PEDs. The exact behav- ior of the stent envelope strongly depends on both of the type of device (De Bock et al., 2012) and physician practice (Ma et al., 2014) which can be precisely modeled only by cumbersome FEA. Moreover, the vasculature modifications which frequently occur during the procedures or resulting from the stent-artery interaction (De Bock et al., 2012) are very difficult to predict since they depend on the surrounding tissues. In the proposed model, both of the patient and clinical practice variability have been neglected assuming an ideal tubular deployment inscribed in the vessel lumen.

The smoothed vessel radius, taken as the radius of the virtual stent envelope, matched very well the dimensions of the real stent cross-section in both the in-vitro and in-vivo comparisons. However, moderate dispersion of the virtual deployment radius was observed compared to real stent radius. The precision of both the acquisition modality and the segmentation process are therefore critical to ensuring a reliable prediction. In the present work, we used the most precise clinical modality i.e. 3DRA for the vasculature imaging. The strong contrast between vasculature and surrounding tissues along with interactive watershed segmentation ensured a robust lumen extraction.

In order to avoid stent protrusion in the aneurysmal cavity, the vessel centerline and radius were both regularized after removing the centerline section at the neck level. Although these regularizations allowed to reproduce the smooth stent behavior at IA neck as observed in patients, they could also lead to small protrusion of the stent through the vessel surface. Therefore, additional inflation of the vessel shape are locally and slightly required to avoiding wrong meshing issues in further CFD investigations.

\subsection{Clinical discussion}

During the last decades, stent assisted coiling has extended the use of endovascular treatments to brain aneurysms with good associated outcomes in term of stabilization of the aneurysmal lesion (King et al., 2014). However, some papers have reported complications related to suboptimal deployments of intracranial stent. For instance, incomplete stent apposition (ISA) to the vessel wall was reported in 19 over 39 patients implanted with Enterprise stents (Heller and Malek, 2011). They showed that ISA was related to geometrical features of the vessel but also to the stent-subtended angle within the curve. Therefore, the use of our model for selecting the landing zone which minimizes this angle could potentially solve this issue.

More recently, FDSs have revolutionized the treatment of un-coilable sidewall aneurysms (Byrne et al., 2010; Becske et al., 2013) localized at the internal carotid artery in the ophtalmic and paraclinoidian segments. Their use has rapidly increased and new indications e.g. fusiform and bifurcation aneurysms (Toth et al., 2014) are now explored. FDS treatment is associated with middle term 
complete resolution outcomes of $76 \%$ at 6 months (Brinjikji et al., 2013) with a relatively low rate of complications even though they remain severe (Kulcsar et al., 2011) (delayed rupture, side branch occlusion). Nevertheless, the secure FDS deployment manoeuvre ensuring an optimal apposition of the device to the vessel wall throughout the arterial curves requires from the operator particular skills and training. Raymond et al. extensively studied the behavior of FDSs in various in-vitro and in-vivo experiments to confront deployment issues and intra-aneurysmal thrombus formation (Darsaut et al., 2013, 2012; Bing et al., 2013). In particular, they described accordion effect which induced ISA due to related proximal spindleshaped deformation of the stent (cigare effect). It was shown that the size of the device and the length of the landing zone strongly influenced this stenosis of the stent extremities. Furthermore, the clinician has to avoid the coverage of side branches (Lescher et al., 2014) to prevent ischemic events as reported in FDS treatment of basilar artery aneurysms (Kulcsar et al., 2010). Consequently, the device sizing and its accurate positioning are essential factors considered in the overall treatment strategy.

However, the position of the stent is very difficult to estimate from the manufacturers tables providing mainly cylindrical deployment details due to the variability of the vessel shape and diameter. For instance large differences in vessel diameter frequently occur between the proximal/distal segments of the parent arteries. These preliminary results show the potential of our geometrical model to give such crucial information for any kind of braided stents. Its low computation cost and required manual operation make the implementation of an interactive tool highly feasible. Therefore, the clinician could test different stent configurations to choose the most appropriate device prior to the implantation procedure. Furthermore, the available local porosity would provide refined selection criteria to the clinician complementary to CFD evaluation. Indeed, it has been shown that the intra-aneurysmal flow reduction depends on the device porosity (Mut and Cebral, 2012). Additional studies are ongoing to test the predictions of the deployment model on a large sample of patients and different types of FDSs.

\section{Conclusion}

In this study, we proposed a new mathematical model for braided stent deployment. Based on few geometrical constraints and parameters this model predicted the wires location as well as the length and local porosity of the stent for the first time. Measurements of in-vitro and invivo FDS deployments confirmed qualitatively and quantitatively the high accuracy of the proposed model and its underlying assumptions. Thanks to its minimal computational time, this model is easily implementable in a clinical tool for providing critical per-operative information to the clinician.

\section{Acknowledgment}

This study was supported by Swiss National Science Foundation grants (SNF 32003B_160222 and SNF 320030_156813). We would like to thank D. Baer for the pictures of the in-vitro stent implantations.

\section{References}

Antiga, L., Piccinelli, M., Botti, L., Ene-Iordache, B., Remuzzi, A., Steinman, D. A., 2008. An image-based modeling framework for patient-specific computational hemodynamics. Med Biol Eng Comput 46 (11), 1097-1112.

Appanaboyina, S., Mut, F., Loehner, R., Putman, C., Cebral, J., 2009. Simulation of intracranial aneurysm stenting: Techniques and challenges. Comput Method Appl M 198 (45-46), 3567-3582.

Appanaboyina, S., Mut, F., Lohner, R., Putman, C. A., Cebral, J. R., 2008. Computational fluid dynamics of stented intracranial aneurysms using adaptive embedded unstructured grids. Int J Numer Meth 57 (5), 475-493.

Becske, T., Kallmes, D. F., Saatci, I., McDougall, C. G., Szikora, I., Lanzino, G., Moran, C. J., Woo, H. H., Lopes, D. K., Berez, A. L., Cher, D. J., Siddiqui, A. H., Levy, E. I., Albuquerque, F. C., Fiorella, D. J., Berentei, Z., Marosfoi, M., Cekirge, S. H., Nelson, P. K., 2013. Pipeline for uncoilable or failed aneurysms: Results from a multicenter clinical trial. Radiology 267 (3), 858-868.

Bernardini, A., Larrabide, I., Petrini, L., Pennati, G., Flore, E., Kim, M., Frangi, A. F., 2012. Deployment of self-expandable stents in aneurysmatic cerebral vessels: comparison of different computational approaches for interventional planning. Comput Methods Biomech Biomed Engin 15 (3), 303-311.

Bing, F., Darsaut, T. E., Salazkin, I., Makoyeva, A., Gevry, G., Raymond, J., 2013. Stents and flow diverters in the treatment of aneurysms: device deformation in vivo may alter porosity and impact efficacy. Neuroradiology 55 (1), 85-92.

Bouillot, P., Brina, O., Ouared, R., Lovblad, K. O., Farhat, M. Mendes Pereira, V., 2014a. Particle imaging velocimetry evaluation of intracranial stents in sidewall aneurysm: hemodynamic transition related to the stent design. PLOS ONE 9, e113762.

Bouillot, P., Brina, O., Ouared, R., Lovblad, K. O., Mendes Pereira, V., Farhat, M., 2014b. Multi-time-lag PIV analysis of steady and pulsatile flows in a sidewall aneurysm. Exp Fluids 55 (6), 1746.

Brinjikji, W., Murad, M. H., Lanzino, G., Cloft, H. J., Kallmes, D. F., 2013. Endovascular treatment of intracranial aneurysms with flow diverters a meta-analysis. Stroke 44 (2), 442-447.

Byrne, J. V., Beltechi, R., Yarnold, J. A., Birks, J., Kamran, M., 2010. Early experience in the treatment of intra-cranial aneurysms by endovascular flow diversion: A multicentre prospective study. PLOS ONE 5 (9), e12492.

Chong, W., Zhang, Y., Qian, Y., Lai, L., Parker, G., Mitchell, K., 2013. Computational hemodynamics analysis of intracranial aneurysms treated with flow diverters: Correlation with clinical outcomes. AJNR Am J Neuroradiol 35 (2), 136-142.

Darsaut, T. E., Bing, F., Makoyeva, A., Gevry, G., Salazkin, I., Raymond, J., 2013. Flow diversion to treat aneurysms: the free segment of stent. J Neurointerv Surg 5 (5), 452-457.

Darsaut, T. E., Bing, F., Salazkin, I., Gevry, G., Raymond, J., 2012. Flow diverters can occlude aneurysms and preserve arterial branches: A new experimental model. AJNR Am J Neuroradiol 33 (10), 2004-2009.

De Bock, S., Iannaccone, F., De Santis, G., De Beule, M., Mortier, P., Verhegghe, B., Segers, P., 2012. Our capricious vessels: The influence of stent design and vessel geometry on the mechanics of intracranial aneurysm stent deployment. J Biomech 45 (8), 1353 1359. 
Florez Valencia, L., Montagnat, J., Orkisz, M., 2004. 3D graphical models for vascular-stent pose simulation. MGV 13 (3), 235-248.

Gascou, G., Lobotesis, K., Brunel, H., Machi, P., Riquelme, C., Eker, O., Bonafe, A., Costalat, V., 2015. Extra-aneurysmal flow modification following pipeline embolization device implantation: Focus on regional branches, perforators, and the parent vessel. AJNR Am J Neuroradiol 36 (4), 725-731.

Heller, R. S., Malek, A. M., 2011. Parent vessel size and curvature strongly influence risk of incomplete stent apposition in Enterprise intracranial aneurysm stent coiling. AJNR Am J Neuroradiol 32 (9), 1714-1720.

Higgins, W., Ojard, E., 1993. Interactive morphological watershed analysis for 3D medical images. Comput Med Imaging Graph 17 (4-5), 387-395.

Janiga, G., Roessl, C., Skalej, M., Thevenin, D., 2013. Realistic virtual intracranial stenting and computational fluid dynamics for treatment analysis. J Biomech 46 (1), 7-12.

Jedwab, M., Clerc, C., 1993. A study of the geometrical and mechanical-properties of a self-expanding metallic stent theory and experiment. J Appl Biomater 4 (1), 77-85.

Kim, J. H., Kang, T. J., Yu, W.-R., 2008. Mechanical modeling of self-expandable stent fabricated using braiding technology. J Biomech 41 (15), 3202-3212.

King, B., Vaziri, S., Singla, A., Fargen, K., Mocco, J., 2014. Clinical and angiographic outcomes after stent-assisted coiling of cerebral aneurysms with Enterprise and Neuroform stents: a comparative analysis of the literature. J Neurointerv Surg, in press.

Kulcsar, Z., Augsburger, L., Reymond, P., Pereira, V. M., Hirsch, S., Mallik, A. S., Millar, J., Wetzel, S. G., Wanke, I., Ruefenacht, D. A., 2012. Flow diversion treatment: intra-aneurismal blood flow velocity and wss reduction are parameters to predict aneurysm thrombosis. Acta Neurochir 154 (10), 1827-1834.

Kulcsar, Z., Ernemann, U., Wetzel, S. G., Bock, A., Goericke, S., Panagiotopoulos, V., Forsting, M., Ruefenacht, D. A., Wanke, I., 2010. High-profile flow diverter (Silk) implantation in the basilar artery efficacy in the treatment of aneurysms and the role of the perforators. Stroke 41 (8), 1690-1696.

Kulcsar, Z., Houdart, E., Bonafe, A., Parker, G., Millar, J., Goddard, A. J. P., Renowden, S., Gal, G., Turowski, B., Mitchell, K., Gray, F., Rodriguez, M., van den Berg, R., Gruber, A., Desal, H., Wanke, I., Ruefenacht, D. A., 2011. Intra-aneurysmal thrombosis as a possible cause of delayed aneurysm rupture after flowdiversion treatment. AJNR Am J Neuroradiol 32 (1), 20-25.

Lagarias, J., Reeds, J., Wright, M., Wright, P., 1998. Convergence properties of the Nelder-Mead simplex method in low dimensions. Siam J Numer Anal 9 (1), 112-147.

Larrabide, I., Geers, A. J., Morales, H. G., Bijlenga, P., Rüfenacht, D. A., 2015. Change in aneurysmal flow pulsatility after flow diverter treatment. Comput Med Imag Grap, in press.

Larrabide, I., Kim, M., Augsburger, L., Cruz Villa-Uriol, M., Ruefenacht, D., Frangi, A. F., 2012. Fast virtual deployment of selfexpandable stents: Method and in vitro evaluation for intracranial aneurysmal stenting. Med Imag Anal 16 (3), 721-730.

Lescher, S., Samaan, T., Berkefeld, J., 2014. Evaluation of the pontine perforators of the basilar artery using digital subtraction angiography in high resolution and $3 \mathrm{D}$ rotation technique. AJNR Am J Neuroradiol 35 (10), 1942-1947.

Ma, D., Dargush, G. F., Natarajan, S. K., Levy, E. I., Siddiqui, A. H., Meng, H., 2012. Computer modeling of deployment and mechanical expansion of neurovascular flow diverter in patientspecific intracranial aneurysms. J Biomech 45 (13), 2256-2263.

Ma, D., Dumont, T. M., Kosukegawa, H., Ohta, M., Yang, X., Siddiqui, A. H., Meng, H., 2013. High fidelity virtual stenting (HiFiVS) for intracranial aneurysm flow diversion: In vitro and in silico. Ann Biomed Eng 41 (10), 2143-2156.

Ma, D., Xiang, J., Choi, H., Dumont, T. M., Natarajan, S. K., Siddiqui, A. H., Meng, H., 2014. Enhanced aneurysmal flow diversion using a dynamic push-pull technique: An experimental and modeling study. AJNR Am J Neuroradiol 35 (9), 1779-1785.

Meyer, F., 1994. Topographic distance and watershed lines. Signal Processing 38 (1), 113-125.
Murthy, S. B., Shah, S., Rao, C. P. V., Bershad, E. M., Suarez, J. I., 2014. Treatment of unruptured intracranial aneurysms with the pipeline embolization device. J Clin Neurosci 21 (1), 6-11.

Mut, F., Cebral, J. R., 2012. Effects of flow-diverting device oversizing on hemodynamics alteration in cerebral aneurysms. AJNR Am J Neuroradiol 33 (10), 2010-2016.

Mut, F., Raschi, M., Scrivano, E., Bleise, C., Chudyk, J., Ceratto, R., Lylyk, P., Cebral, J. R., 2015. Association between hemodynamic conditions and occlusion times after flow diversion in cerebral aneurysms. J Neurointerv Surg 7 (4), 286-290.

Pereira, V., Brina, O., Delattre, B., Ouared, R., Bouillot, P., Erceg, G., Schaller, K., Lovblad, K., Vargas, M., 2014a. Assessment of intra-aneurysmal flow modification after flow diverter stent placement with four-dimensional flow MRI: a feasibility study. J Neurointerv Surg, in press.

Pereira, V. M., Brina, O., Bijlenga, P., Bouillot, P., Schaller, K., Lovblad, K.-O., Ouared, R., 2014b. CFD analysis of flow reduction induced by low porosity stent in intracranial aneurysms: the patient-unspecific flow change perspective. submitted to J Neurointerv Surg.

Shapiro, M., Raz, E., Becske, T., Nelson, P. K., 2014. Variable porosity of the pipeline embolization device in straight and curved vessels: A guide for optimal deployment strategy. AJNR Am J Neuroradiol 35 (4), 727-733.

Spranger, K., Ventikos, Y., 2014. Which spring is the best? Comparison of methods for virtual stenting. IEEE Trans Biomed Eng 61 (7), 1998-2010.

Toth, G., Bain, M., Hussain, M., Moskowitz, S., Masaryk, T., Rasmussen, P., Hui, F., 2014. Posterior circulation flow diversion: a single-center experience and literature review. J Neurointerv Surg 7 (8), 574-583.

Turjman, F., Levrier, O., Combaz, X., Bonafe, A., Biondi, A., Desal, H., Bracard, S., Mounayer, C., Riva, R., Chapuis, F., Huot, L., Armoiry, X., Gory, B., 2015. Evidence trial: design of a phase 2, randomized, controlled, multicenter study comparing flow diversion and traditional endovascular strategy in unruptured saccular wide-necked intracranial aneurysms. Neuroradiology 57 (1), 4954.

Wetzel, S., Ohta, M., Handa, A., Auer, J., Lylyk, P., Lovblad, K., Babic, D., Rufenacht, D., 2005. From patient to model: Stereolithographic modeling of the cerebral vasculature based on rotational angiography. AJNR Am J Neuroradiol 26 (6), 1425-1427.

Zanaty, M., Jabbour, P. M., Sader, R. B., Chalouhi, N., Tjoumakaris, S., Rosenwasser, R. H., Gonzalez, L. F., 2014. Intra-aneurysmal thrombus modification after flow-diversion. J Clin Neurosci 22 (1), 105-110. 